\title{
Evaluation of periodontal condition in individuals with down syndrome: a case-control study
}

\begin{abstract}
Ogival palate, macroglossia, anodontia, cleft tongue, delayed tooth eruption, low prevalence of caries and high prevalence of periodontal disease are the main oral manifestations of Down syndrome. The aim of the present observational study was to evaluate the periodontal condition in individuals with Down syndrome. The study included 30 individuals who were selected according to the age group to be studied, between 17 and 25 years old, in a local institution for the care of Down syndrome (DG) patients and 30 individuals who made up the control group and who did not have Down syndrome (CG), periodontal parameters were observed in both groups that were adjusted for habits. The results showed for the Plaque Index $41.2 \%$ of the individuals in the DS group while in the CG group in $45.5 \%$ without statistical difference. For the Gingival Index, it was observed that $39.7 \%$ in the DS group and in the CG group $44.2 \%$ without statistical difference between the groups $(\mathrm{p}<0.05)$. Family and institutional participation collaborates in the daily control of dental biofilm. In the present study, individuals in the DG group presented periodontal clinical parameters compatible with periodontal health and when compared to healthy individuals, they did not present clinical differences.
\end{abstract}

Volume 12 Issue 5 - 202I

\begin{abstract}
Caio Vinicius G. Roman-Torres,' Humberto Oswaldo Schwartz-Filho, ${ }^{2}$ Mariana Oliveira, ${ }^{2}$ Camila B. Barbieri,', Marcelo M. Quintela,' Wilson R. Sendyk

'Department of Dentistry, University Santo Amaro/UNISA, Brazil

${ }^{2}$ Department of Stomatology, Federal University of Parana (UFPR), Brazil
\end{abstract}

\author{
Correspondence: Caio Vinicius G. Roman-Torres, Rua \\ Professor Eneas de Siqueira Neto, 340, São Paulo, SP, Brazil, \\ Tel +55 I | 214 I8555, Email cvgrt@hotmail.com
}

Received: March 26, 202I | Published: October 27, 2021

Keywords: periodontitis, periodontal index, down syndrome

\section{Introduction}

In 1866, John Langdon Down noticed different physiognomic characteristics among certain children with mental retardation. Jérôme Lejeune in 1958, noticed an error in the distribution of the cells and they had 47 chromosomes (different from a chromosomally normal individual who has 46 chromosomes), and this chromosome the most linked to pair $21 .^{1}$

Ogival palate, macroglossia, anodontia, cleft tongue, delayed tooth eruption, low prevalence of caries and high prevalence of periodontal disease are the main oral manifestations of Down syndrome. ${ }^{2}$ Periodontal disease is a disease characterized by a set of inflammatory conditions, of chronic character, and of bacterial origin, that begins affecting the gingival tissue and with time the supporting tissues. The microorganisms responsible for these events are present in the dental biofilm. The treatment of this disease is basically characterized by subgingival or supragingival scraping and in more advanced cases there is a need for surgery, therapies and antibiotics.

The mechanical and chemical control of dental biofilm in DS patients using chlorhexidine and erythrosine has been shown to be effective, ${ }^{4}$ but prolonged use can cause adverse effects. The oral hygiene instruction given to parents or guardians of DS, and supragingival and subgingival scraping provided every 6 months by a dentist does not stop the progression of periodontal disease. ${ }^{5,6}$ Studies have shown an increase in periodontitis in DS individuals, with higher levels of plaque index, probing depth and insertion loss and report that the clinical signs of periodontal disease are more severe in individuals with DS. ${ }^{8,9}$ In a recent study, similar periodontal conditions were observed among individuals with and without Down syndrome. ${ }^{10}$ The specific associations between certain subgingival bacterial species and loss of periodontal insertion show higher levels in individuals with DS. ${ }^{2}$ The deficient immune response increases the prevalence and severity of periodontitis in individuals with DS. The prevalence of periodontal disease in adolescents with DS is from $30 \%$ to $40 \%$, and in individuals close to thirty years of age, this percentage rises to around $100 \%$. Individuals with intellectual impairment have poor oral hygiene, due to their difficulty in brushing and this is an important factor for the onset of periodontal disease. ${ }^{11}$

However, poor oral hygiene is not the only explanation for the severe periodontal destruction that occurs in individuals with DS, as it was observed that the prevalence of periodontal disease was higher in children with DS than in children with similar mental retardation. ${ }^{12}$

Children with DS are more likely to have gingival inflammation. ${ }^{13}$ In adults, surgical and non-surgical periodontal therapies after a year show similar clinical improvement. ${ }^{14}$ Control of the supragingival plaque with pharmacological help prevents periodontal destruction. ${ }^{15}$ The need for dental treatment is more elevation of individuals with DS and require instructions on oral hygiene, prophylaxis and complex and continuous treatment. The aim of the present observational study was to evaluate the periodontal condition in individuals with Down syndrome compared to patients without syndrome regarding periodontal clinical parameters.

\section{Methodology}

This study was submitted and approved by the Research Ethics Committee receiving approval number 1.334.359, all individuals or their responsible persons involved received and signed the consent form to participate in the study. The study included 30 individuals who were selected according to the age group to be studied, between 17 and 25 years old, in a local institution for the care of Down syndrome (DG) patients and 30 individuals who made up the control group and who did not have Down syndrome (CG). Patients who used mouthwashes, systemic medication, manual limitation to the use of a manual brush and inability to use dental floss were excluded from the study. 


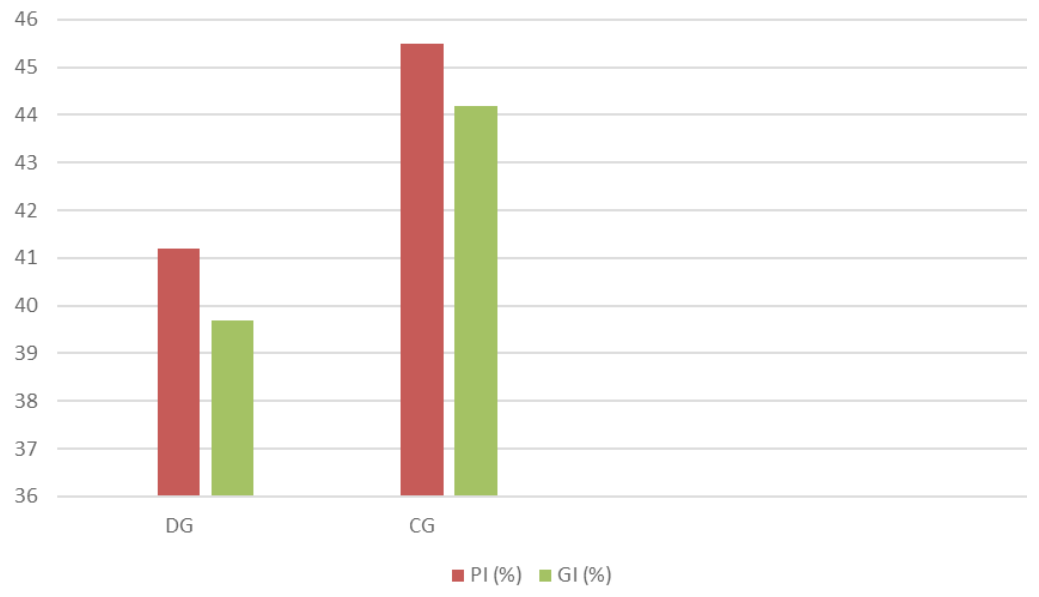

Figure I Plaque and Ginival Index.

PI, Plaque Index; GI, Gingival Index; DG, Down Syndrome Group; CG, Control Group.

Table I Periodontal evaluation divided into two groups

\begin{tabular}{llllll}
\hline & Gender & Age & PD & PI & Gl \\
\hline DG Group & $14 \mathrm{M} / 16 \mathrm{~F}$ & 21,7 & $2,57 \mathrm{~mm}$ & $41,2 \%$ & $39,7 \%$ \\
CG Group & $16 \mathrm{M} / 14 \mathrm{~F}$ & 20,9 & $2,62 \mathrm{~mm}$ & $45,5 \%$ & $44,2 \%$ \\
& & & $\mathrm{p}=0.18$ & $\mathrm{P}=0,08$ & $\mathrm{p}=0,07$
\end{tabular}

PD, Probing Depth; PI, Plaque Index; GI, Gingival Index; DG, Down Syndrome Group; CG, Control Group

The periodontal examination was performed only once by a single operator, previously calibrated. For periodontal evaluation, a millimeter-type Willians (Golgran $\AA$ ) periodontal probe was used. The following were evaluated: probing depth, plaque index and the bleeding index of 4 different sites divided by the buccal, mesial, distal and palatal/lingual surfaces. All participants received instruction in oral hygiene, with recreational activities that sought to involve the participants in order to perform the brushing in front of the researchers so that any wrong performance was immediately corrected. All participants were instructed to brush their teeth at least twice a day and floss once.

After tabulation of the clinical data of the patients included in the present study, they were subjected to specific statistical analysis. For that, the SPSS 27.0 and Bioestat 5.0 softwares were used. In all analytical situations, a $95 \%$ statistical significance level $(\mathrm{p} \leq 0.05)$ was adopted. For each analytical group of interest, the sample distribution characteristic was tested and the statistical test selected using the two way Anova tests and the Test-t for paired samples.

\section{Results}

The individuals were divided into two groups, DS composed of 14 males and 16 females and with a mean age of 21.7 years. The control group (CG) included chromosomally normal individuals, 14 males and 16 females with a mean age of 20.9 years. Among the clinical parameters evaluated, when observing the probing depth (PD), the DS group had an average of $2.57 \mathrm{~mm}$ while the CG group had an average of $2.62 \mathrm{~mm}$, with no statistical difference between the groups.

For the Plaque Index (PI), the presence of dental biofilm was observed in $41.2 \%$ of the individuals in the DS group while in the CG group in $45.5 \%$ of the individuals, but without statistical difference. For the Gingival Index (GI), it was observed that $39.7 \%$ of the individuals in the DS group had bleeding on probing and in the CG group $44.2 \%$ had bleeding, with no statistical difference between the groups $(\mathrm{p}<0.05)$.

\section{Discussion}

Down syndrome is a genetic disease known as trisomy 21 , the condition is associated with an extra chromosome, an estimated prevalence of 9.2 cases per 10,000 live births. ${ }^{1}$ The oral conditions of individuals with Down syndrome, such as chronic periodontitis and aggressive periodontitis, are characterized by a progressive gingival inflammatory response to bacteria in the dental plaque, leading to the destruction of the periodontium. ${ }^{3}$ Periodontitis has an earlier onset and presents a more severe generalized progression than in people without systemic involvement. ${ }^{8,9,13}$

Patients with Down syndrome have a higher prevalence of periodontal disease, which are factors, progression and dissemination of periodontal pathogens in the bloodstream, which may predispose these patients to chronic systemic diseases. ${ }^{4,6}$ Mechanical control produces significant reductions in gingivitis in people with special needs. However, many patients with Down syndrome, in addition to being unable to cooperate, do not have enough manual dexterity to brush or floss. The mechanical and chemical control of biofilm in patients with Down syndrome, using different toothpastes. The plaque developer significantly decreased the plaque index, but the combination containing chlorhexidine and erythrosine gave better results. $^{5}$

Patients with Down's syndrome were compared with patients with cerebral palsy. Clinical rates of periodontal inflammation and treatment needs were statistically significantly higher among patients with Down syndrome. ${ }^{11}$ An increase in periodontitis with higher levels of plaque index, probing depth and loss of insertion where clinical signs of periodontal disease was observed in normal individuals when compared to individuals with Down syndrome. ${ }^{7,10}$ 
The severe periodontal destruction could not be explained by oral problems, only hygiene. Several laboratory studies have demonstrated reduced chemotaxis, decreased phagocytic capacity, defective oxidative response and changes in leukocyte bactericidal activity. ${ }^{4} \mathrm{~A}$ study was carried out with patients who live in institutions or with their parents. It was possible to conclude through this study that patients who live in homes with their parents have better periodontal conditions when compared to patients who live in institutions. ${ }^{15}$

In our study, when probing depth and plaque and bleeding rates were found, clinical differences were found with better rates for individuals in the test group, but when statistically assessed, no differences were observed. Some authors have evaluated that, in a comparative picture between chromosomally normal individuals and patients with Down Syndrome in the face of periodontal treatment, the groups of patients with DS demonstrated significantly higher prevalence and severity of periodontal disease in all items evaluated such as: bleeding, periodontal pocket index and bacterial plaque. ${ }^{2}$

Our study shows clinical differences with others in the literature due to the fact that these individuals with Down syndrome have full treatment throughout the day in the institutions, parents are often alerted about oral problems and always attend lectures on oral care that are offered annually. Past information is applied both at school and at home. A study that included plaque and gingival index as clinical parameters, after scaling and root planing, $1 \%$ chlorhexidine was used daily, the results improved a lot with respect to the plaque index. $^{6}$

Some people with Down syndrome can brush and floss independently, but many need help. Talk to their caregivers about daily oral hygiene. Do not assume that all caregivers know the basics; demonstrate proper brushing and flossing techniques. A power toothbrush or a floss holder can simplify oral care. Also, use your experiences with each patient to demonstrate sitting or standing positions for the caregiver. Emphasize that a consistent approach to oral hygiene is important - caregivers should try to use the same location, timing, and positioning.

Patients with Down syndrome with hyposalivation are at increased risk for the development of periodontal disease. Poor oral hygiene does not automatically mean that periodontitis will develop, but when susceptibility to periodontitis has been demonstrated, good oral hygiene helps to reduce and control periodontitis. ${ }^{5}$ To prevent gum disease brush twice daily, focusing the bristles along the gum line, floss daily and be sure to visit the dentist regularly to have gum health monitored and to take X-rays to monitor bone levels. If the gums bleed that means that they are inflamed. Brushing and flossing should not be stopped because of this. In fact, brushing and flossing will keep the gums clean and help to minimize the inflammation.

Poor oral hygiene does not automatically mean that periodontitis will develop, but when susceptibility to periodontitis has been demonstrated, good oral hygiene helps to reduce and control periodontitis. ${ }^{2}$ The observed results show a better periodontal clinical condition of individuals with Down syndrome, due to the fact that individuals with Down syndrome are subjected to daily care. Oral hygiene instructions and daily tooth brushing were observed, which probably resulted in the clinical parameters observed in the individuals.

\section{Conclusion}

In the present study, individuals in the DG group presented periodontal clinical parameters compatible with periodontal health and when compared to healthy individuals, they did not present clinical differences. Family and institutional participation collaborates in the daily control of dental biofilm.

\section{Acknowledgments}

None.

\section{Conflicts of interest}

None.

\section{References}

1. Frydman A, Nowzari H. Down syndrome-associated periodontitis: a critical review of the literature. Compend Contin Educ Dent. 2012;33(5):356-61.

2. Babette VW, van Loon M, Reuland W, Bruers J. Periodontal disease in Down's syndrome patients. A retrospective study. Spec Care Dentist. 2018;1-8.

3. Cavalcante LB, Tanaka MH, Pires JR, et al. Expression of the Interleukin-10 Signaling Pathway Genes in Individuals With Down Syndrome and Periodontitis. J Periodontol. 2012;83:926-935.

4. Cichon P, Crawford L, Grimm WD. Early-onset periodontitis associated with Down's syndrome - clinical interventional study. Ann Periodontol. 1998;3(1):370-380.

5. Culebras-Atienza E, Silvestre FJ, Silvestre-Rangil J. Possible association between obesity and periodontitis in patients with Down syndrome. Med Oral Patol Oral Cir Bucal. 2018;1;23(3):e335-343.

6. Ferreira R, Michel RC, Greghi SL, et al. Preventative therapies and periodontal interventions for Down syndrome patients. Evidence-Based Dentistry. 2016;17:101-102.

7. Khocht A, Janal M, Turner B. Periodontal health in Down syndrome: Contributions of mental disability, personal, and professional dental care. Spec Care Dentist. 2010;30(3):118-123.

8. Zaldivar-Chiapa RM, Arce-Mendoza AY, De La Rosa-Ramírez M, et al. Evaluation of surgical and non-surgical periodontal therapies, and immunological status, of young Down's syndrome patients. J Periodontol. 2005;76(7):1061-1065.

9. Nuernberg MAA, Ivanaga CA, Haas AN, et al. Periodontal status of individuals with Down syndrome: sociodemographic, behavioural and family perception influence. Journal of Intellectual Disability Research. 2019;1-12.

10. Ribeiro RA, Nascimento LA, Torres CVGR, et al. Periodontal clinical evaluation in individuals with Down Syndrome. Braz J Periodontol. 2016; 26(2):23-27.

11. Sakellari D, Arapostathis KN, Konstantinidis A. Periodontal conditions and subgingival microflora in Down syndrome patients. A case-control study. J Clin Periodontol. 2005;32:684-690.

12. Souza RC, Giovani EM, Élcio Magdalena Giovani. Condiciones periodontales asociadas con hiposalivacíon en pacientes con síndrome de Down. Odontología. 2018;20(1):75-87.

13. Tanaka MH, Rodrigues TO, Finoti LS, et al. Salivary Periodontopathic Bacteria in Children and Adolescents with Down Syndrome. Eur J Clin Microbiol Infect Dis. 2015;34:601-608.

14. Czlusniak GD. Evaluation of the mechanical and chemical control of dental biofilm in patients with Down syndrome. Community Dent Oral Epidemiol. 2009;37:463-467.

15. Zizzi A, Piemontese M, Gesuita R, et al. Periodontal status in the Down's syndrome subjects living in central-eastern Italy: the effects of place of living. Int J Dent Hygiene. 2014;12:193-198. 